\title{
Performance Analysis of Optical Amplifiers (EDFA and SOA)
}

\author{
Nabil Elsheikh Mohamed Elmak ${ }^{1}$, Dr. Amin Babiker A/Nabi Mustafa ${ }^{2}$ \\ ${ }^{1,2}$ (Telecommunications Engineering, Faculty of Engineering/Neelain University, Sudan)
}

\begin{abstract}
In the optical transmission systems attenuation causes signal power to drop through an optical fiber link, so need to use amplifiers to increase signal power with low noise. Semiconductor Optical Amplifier (SOA) and Erbium-Doped Fiber Amplifier (EDFA) are two of the main types of optical amplifiers, and they were used in this simulation model to analyze their performance, with a data rate of $622 \mathrm{Mb} / \mathrm{s}$ (STM-4 level) and $170 \mathrm{~km}$ optical fiber length for each simulation model. This was simulated by using OptiSystem simulator, including the main parameters of the optical transmission system as input power $(\mathrm{dBm})$, optical fiber cable length $(\mathrm{km})$ and attenuation per length of optical fiber cable $(\mathrm{dB} / \mathrm{km})$, also there are three parameters will be considered which they are output power (dBm), Q-Factor and Bit Error Rate (BER) at receiver, and also Eye Diagram.
\end{abstract}

Keywords: EDFA, Optical Amplifier, Optisystem simulator, Optical Transmission System, SOA

\section{Introduction}

The simple optical transmission system consists of three necessary elements which are fiber cable (transmission channel), light sources as the input and light detector as the output [1]. Also, there are optical amplifiers a key component in an optical communication system which used to increase the power of the signal and reduce the attenuation. Optical amplifiers perform a most important role in modern optical networks, allowing the transmission of highest data rates over long distance without attenuation [5].

There are multiple types of optical amplifiers and the most common used in the optical transmission system are Semiconductor Optical Amplifier (SOA) and Erbium Doped Fiber Amplifier (EDFA). SOA amplifiers use a semiconductor to provide the gain medium, the structure of SOA is similar to semiconductor lasers. They consist of an active part and a passive part made of a semiconductor material such as indium phosphide; it has used as discrete amplifiers [2]. SOAs are fabricated with layers of antireflection coats to avoid light from reflecting into the circuit. Optical gain takes place when current has directed across the p-n junction, this process causes the photons to duplicate, generating signal gain [3].

Erbium-doped fiber amplifier (EDFA) have deployed in undersea links and now are considered as standard amplifier using a well- known technology [4]. Commercially available since the early of 1990's, EDFA can amplify a large wavelength range from $1500 \mathrm{~nm}$ to $1600 \mathrm{~nm}$ at the same time with high gain, it consists of a length of Erbium doped fiber, a laser diode (used as a pump) and wavelength selective coupler to combine the signal and pump wavelength [4].

EDFA has three Categories:

1- In-line amplifiers:

Installed between transmitter and receiver.

2- Power Boosters:

Installed after transmitter output.

3- Pre-amplifiers:

Installed in front of the receiver.

This paper briefly discusses the performance of EDFA and SOA in optical transmission systems using simulation approach (Optisystem v7.0). Optisystem software is a modern simulator tool for designing, testing and optimizing of the optical systems. It is used to design and plan the optical systems models and present the analysis and results of scenarios visually. It is based on realistic of optical fiber communication systems [1].

\section{Methodology}

In analyzing and designing optical networks, there are several methods can be used. For this study simulation approach is used. Optisystem software v7.0 was selected to use in designing two optical systems one is using EDFA and SOA for the other one.

Each system has simulated by the same set of parameters, and each system consists of Transmitter, Optical Amplifier (EDFA/SOA), Optical Fiber Cable, Receiver (Photo Detector and LPF), Power Meters and Eye Diagram Analyzer, as shown below in Fig. (1) and Fig. (2). 


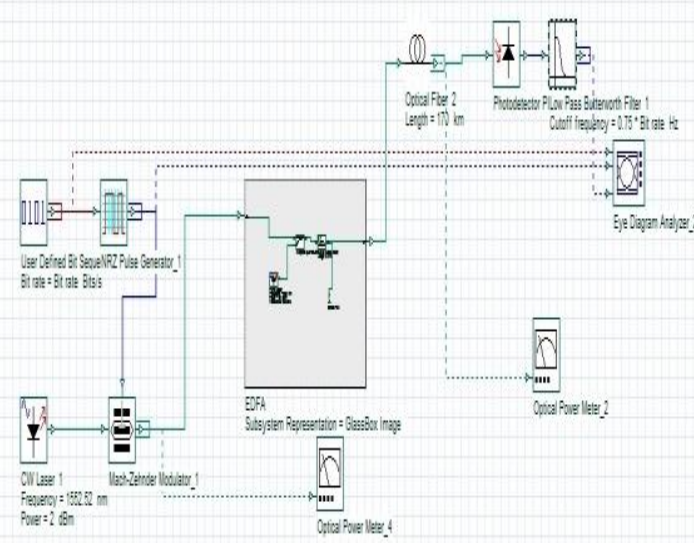

Fig. 1 Optical System Model using EDFA

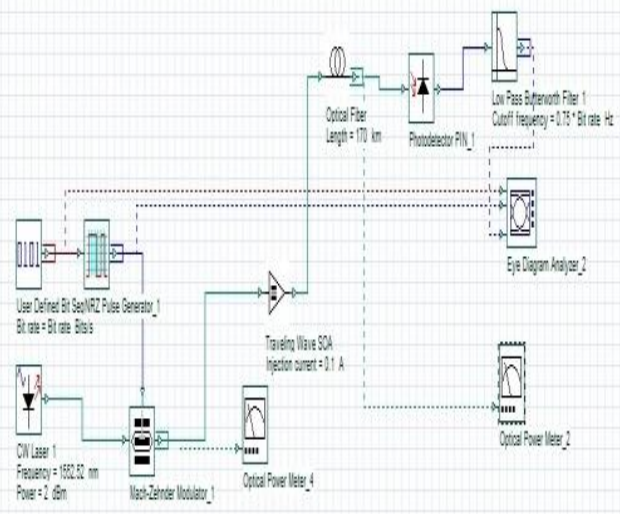

Fig. 2 Optical System Model using SOA

The design parameters are the system parameters that a designer can vary to study their effect on the system's performance. The design parameters consist of a length of Optical Fiber Cable (km), Bit rate (bit/s), Transmit Power $(\mathrm{dBm})$, Loss of Cable $(\mathrm{dB} / \mathrm{km})$ and Frequency $(\mathrm{nm})$ for each optical system model shown in Table (1).

Table 1 The Design Parameters

\begin{tabular}{|l|l|l|}
\hline Description & Values & Unit \\
\hline Optical fiber length & 170 & $\mathrm{Km}$ \\
\hline Bit rate & 622 & $\mathrm{Mb} / \mathrm{s}$ \\
\hline Transmit Power & -0.44 & $\mathrm{dBm}$ \\
\hline Loss of Cable & 0.2 & $\mathrm{~dB} / \mathrm{km}$ \\
\hline Frequency & 1550 & $\mathrm{Nm}$ \\
\hline
\end{tabular}

After run simulation for both optical systems model these parameters (Receive Power, Q-factor, BER and Eye Diagram graph were examined to analyze the performance of EDFA and SOA for designed optical system.

\section{Results and Discussion}

The paper examined three different types of parameters values for both optical system models (Receive Power, Q-factor and Bit Error Rate) throughout the simulation as shown in Table (2). The Eye Diagram graphs also have plotted as shown in Figure (3) and Figure (4).

Table 2 The Examined Parameters

\begin{tabular}{|l|l|l|l|}
\hline Description & Using EDFA & Using SOA & Unit \\
\hline Receive Power & -16.320 & -17.227 & $\mathrm{dBm}$ \\
\hline Q-factor & 33.801 & 11.132 & percentage \\
\hline Bit Error Rate & $9.50984 \mathrm{e}-251$ & $4.3696 \mathrm{e}-29$ & percentage \\
\hline
\end{tabular}

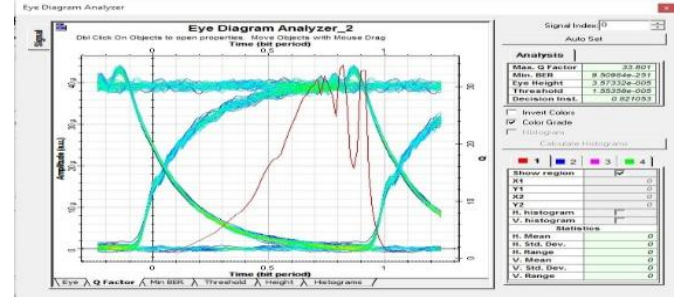

Fig.3 Eye Diagram graph using EDFA

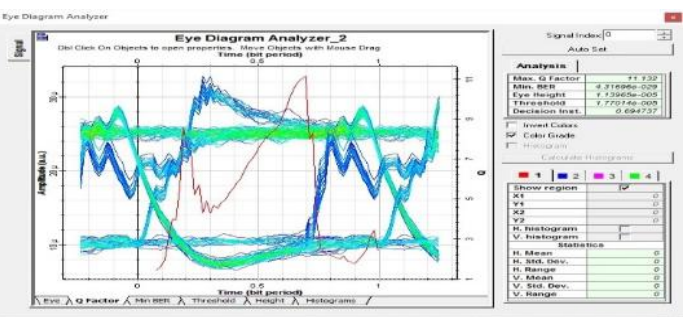

Fig. 4 Eye Diagram graph using SOA

From the simulation results, as shown in Table (2), it shows that power received when EDFA is used as an amplifier in the optical system is more than SOA. Q-factor is used to measure the optical performance with BER. Table (2) also shows the Q-factor and BER readings which obtained by using EDFA and SOA in the optical system model, when EDFA is used the Q-factor is higher than SOA and BER is less than SOA.

As can be seen from Figure (3) in the case of using EDFA shows the largest eye diagram opened wider than as shown in Figure (4) when SOA has used, that demonstrate the quality of signal at the receiver. By matching the results from the simulation of optical system models, it is evident when EDFA has used in an optical system for long distance is much better than SOA using. 


\section{Conclusion}

In this work, Optisystem is used as a simulation tool to design and study optical transmission network model using EDFA and SOA and analyze their performance; the simulative analysis presented a gradual decreasing in BER and increasing in Q-factor when EDFA has used, besides that displayed wide open in Eye Diagram and received high power. By compared and analyzed the two optical system simulation model results, the performance of SOA is dissimilar to EDFA, the SOA has greater noise and worse gain, therefore using EDFA in an optical system which has a long distance between transmitter and receiver is better than using SOA.

\section{References}

[1] Aashima Bhardwaj and Gaurav Soni, Performance Analysis of 20Gbps Optical Transmission System Using Fiber Bragg Grating, International Journal of Scientific and Research Publications, Volume 5, Issue 1, January 2015, ISSN $2250-3153$.

[2] Home Birla Institute of Technology and Science, C10 Optical Amplifiers - Optical Amplifiers Chapter 10, https://www.coursehero.com/file/12879505/C10-Optical-Amplifiers/.

[3] Audra Bond, Chapter 6 Optical Amplifiers, http://slideplayer.com/slide/7680367/.

[4] School of Electronic and Communications Engineering, Unit $1.5 \quad$ Optical Amplifiers, http://www.electronics.dit.ie/staff/tfreir/optical_2/Unit_5.ppt.

[5] Sham Arsenal, Optical Amplifiers -The need, Types, Working Principle and Comparison, http://www.slideshare.net. 